\title{
ESTIMACIÓN DE PÉRDIDAS DE AGUA POR EVAPORACIÓN EN EMBALSES DEL PIRINEO
}

\author{
J. I. LÓPEZ MORENO \\ Instituto Pirenaico de Ecología, CSIC, \\ Campus de Aula Dei, Apdo.202. Zaragoza 50080.
}

Correo electrónico de contacto: nlopez@ipe.csic.es

\begin{abstract}
RESUMEN. En este trabajo se han calculado las tasas de evaporación para láminas de agua en siete estaciones meteorológicas muy próximas a embalses pirenaicos. Los embalses analizados poseen características muy contrastadas en cuanto a su localización, régimen de gestión y relación entre volumen embalsado y superficie inundada. A partir de la estimación de la demanda evaporativa y del conocimiento de la evolución diaria de la superficie inundada por los embalses, se ha realizado un cálculo realista de las pérdidas de agua por evaporación. Los resultados informan de una notable variabilidad en las tasas de evaporación entre los casos de estudio, y fuertes contrastes en las cantidades totales evaporadas, que en ocasiones llegan a alcanzar los 20 hectómetros cúbicos anuales. Se ha podido comprobar que las condiciones climáticas dominantes no son el factor más importante para explicar la magnitud de las pérdidas. Las características morfológicas del vaso de los embalses y el régimen de gestión que se les aplica pueden tener una influencia mayor que las tasas de evaporación estimadas. Aunque en la actualidad, las pérdidas de agua por evaporación no es un problema mayor en la gestión de los recursos hídricos de la región (las pérdidas son siempre inferiores al 6\% de la capacidad de los embalses y del $2 \%$ de los caudales de los ríos), pueden tener mayor importancia en el futuro, en función del aumento global de la temperatura y de una menor disponibilidad de agua.
\end{abstract}

ABSTRACT. This paper estimates the evaporation rates from open water surfaces using data from meteorological stations, which are closely located to seven Pyrenean reservoirs. The studied reservoirs have marked differences in their location characteristics, patterns of management and storage volume-surface flooded relationship. The combination of the evaporative rates with the daily changes on surface flooded by dams enabled us to obtain a realistic assessment of the annual volume of water lost by evaporative processes. Climate is not always the main factor to explain differences in water losses. Reservoirs morphology and the management patterns of the reservoirs may have a larger influ- 
ence than the evaporative rates. Currently, water losses by evaporation do not represent a key-problem in water resource management of the region, since they do not ever exceed a $6 \%$ of the storage capacity, nor a $2 \%$ of river flows. Nevertheless, losses might become a higher problem as temperatures will warm and water resources availability will decline, according to mid-term projections for the region.

Palabras clave: evaporación, embalses, gestión de recursos hídricos, Pirineos. Key words: evaporation, reservoirs, water resources management, Pyrenees.

Enviado el 14 de febrero de 2008 Aceptado el 3 de junio de 2008

\section{Introducción}

Los embalses representan una herramienta fundamental para la gestión de los recursos hídricos. Su importancia es aún mayor en ambientes mediterráneos, donde la relativa escasez de agua, la ocurrencia periódica de sequías y una elevada variabilidad interanual obliga a regular intensamente los ríos para adecuar la estacionalidad en la disponibilidad de los recursos a los momentos de mayor demanda, y almacenar caudales excedentes en periodos húmedos para poder hacer frente a momentos de escasez. Sin embargo, la construcción de presas, la inundación de fondos de valle y la modificación de los regímenes fluviales constituye uno de los mayores impactos del hombre sobre el medio natural (Petts, 1984), resulta muy costoso económicamente y puede desencadenar importantes conflictos sociales (McCully, 2001).

En las últimas décadas se ha despertado un notable interés científico por el estudio de numerosos aspectos relacionados con la gestión de los embalses, sobre todo en lo que se refiere a: i) sus impactos en la ocurrencia de eventos extremos (Zsuffa, 1999; Maingi y Marsh, 2002; López Moreno et al., 2002); ii) alteración de los regímenes fluviales (Higgs y Petts, 1998; Batalla et al., 2004; López Moreno et al., 2004a); iii) tasas de sedimentación en embalses y sus consecuencias geomorfológicas aguas abajo de las presas (Kondolf, 1997; Verstraeten y Poessen, 2000; López Moreno et al., 2003; Snyder et al., 2004); y iv) efectos ecológicos y ambientales resultantes de la construcción de embalses (Thomas, 1996; Richter et al., 1997; Sanz-Montero et al., 1999; Sánchez-Pérez y Trémolières, 2003). Sin embargo, otros aspectos relativos a los embalses tan sólo han sido estudiados de una forma muy marginal. Éste es el caso de la cuantificación de pérdidas por procesos de evaporación de la lámina de agua embalsada, cuestión muy poco referida en la literatura científica, y en ocasiones presentando conclusiones contradictorias. En general, existe una tendencia a considerar este proceso como una pérdida difícilmente cuantificable y que tiende a ser compensada por otras aportaciones que no son tenidas en cuenta, como la llegada de escorrentía directa al vaso del embalse o la lluvia sobre la lámina de agua (Montaseri y Adeloye, 2004). En cambio, otros trabajos apun- 
tan que la evaporación producida en la superficie de un embalse supone una pérdida de agua que puede llegar a representar grandes volúmenes (Fennnessey, 1995). Estimaciones de pérdidas por evaporación se han realizado principalmente en sectores áridos o semiáridos, donde las temperaturas son muy elevadas (Wafa y Labib, 1973; Snoussi et al., 2002). En la Península Ibérica, Martínez Álvarez et al. (2004) estimaron una perdida de $7.96 \mathrm{hm}^{3}$ anuales en los embalses y balsas de la Comunidad de Regantes en el Campo de Cartagena, lo que supone un 6.3\% de los recursos hídricos de que disponen. En un trabajo similar Bengoechea et al. (1991) estimaron que el $17 \%$ del total de agua destinada a usos agrícolas se evapora en balsas de riego en el Campo de Dalias (Almería). Ayala-Carcedo (2000) estimó que la evaporación en embalses y humedales en España supone una pérdida de $1.400 \mathrm{hm}^{3}$ año-1 $^{-1}$, un tercio del consumo urbano total, y apunta que, como consecuencia del cambio climático, estas cifras podrían aumentar entre un 11 y un 40\% (escenario 2060) según las cuencas analizadas.

Este trabajo pretende cuantificar las pérdidas de agua por evaporación en siete embalses localizados en el Pirineo Central, de los que se ha dispuesto de la información diaria de las condiciones climáticas y de la superficie inundada que ocupan. Los embalses presentan unas características muy contrastadas en cuanto a su tamaño-volumen, procesos de llenado y vaciado, y condiciones climáticas, lo que permite discutir el impacto de estos tres elementos en el volumen total evaporado.

\section{Zona de estudio}

Los embalses analizados se localizan en el Pirineo Central español, entre las cuencas altas de los ríos Aragón y Noguera Ribagorzana (Figura 1). Las máximas altitudes se encuentran en las cabeceras de los ríos Gállego, Cinca, Ésera y Noguera Ribagorzana, donde se superan ocasionalmente los $3000 \mathrm{~m}$.

Los ríos pirenaicos cortan de norte a sur un relieve organizado en bandas paralelas, atravesando el sector paleozoico o Pirineo Axial (granitos, calizas, pizarras y arcillas), las Sierras Interiores (calizas y areniscas), el Sector del Flysch, la Depresión Interior Altoaragonesa, modelada en margas eocenas, las molasas prepirenaicas (areniscas y arcillas) y las Sierras Exteriores (calizas y margas) que constituyen el extremo sur de la zona de estudio antes de alcanzar la Depresión del Ebro. Las presas de los embalses pirenaicos tienden a estar apoyadas sobre los materiales más sólidos de las Sierras Interiores, en la Depresión Interior e inmediatamente al norte de las Sierras Exteriores, aprovechando pequeñas depresiones margosas.

El clima de la región tiende a perder sus características oceánicas en sentido nortesur y de oeste a este, adquiriendo progresivamente rasgos más mediterráneo-montanos con tendencia continental. Este cambio supone un descenso de la precipitación y un incremento de las temperaturas, si bien estas últimas están controladas principalmente por la altitud. La precipitación oscila entre más de $2000 \mathrm{~mm}$ anuales en los sectores más 


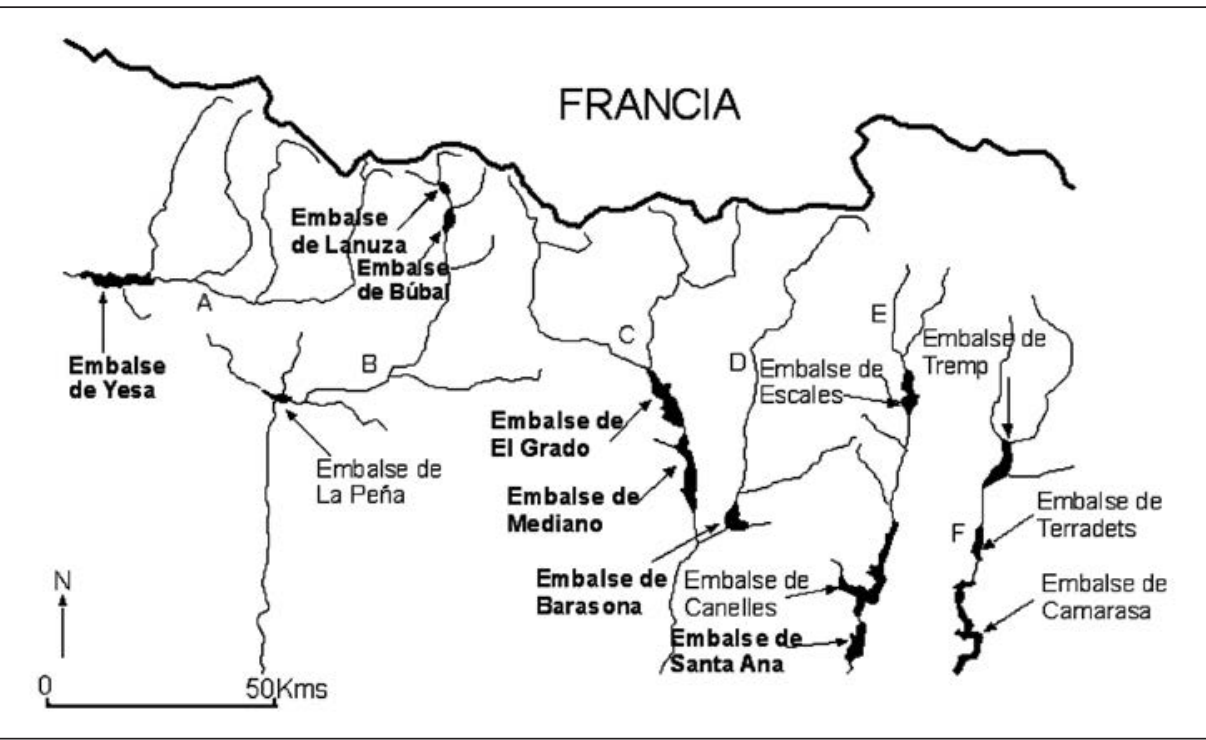

Figura 1. Zona de estudio y sus principales embalses. Los embalses analizados aparecen en negrita. A: río Aragón; B: río Gállego; C: río Cinca; D: río Ésera; E: río N. Ribagorzana; F: río N. Pallaresa

elevados y poco más de $500 \mathrm{~mm}$ al sur de la zona de estudio. Desde diciembre hasta marzo la isoterma de $0{ }^{\circ} \mathrm{C}$ se encuentra en torno a $1600 \mathrm{~m}$ s.n.m. (García Ruiz et al., 1985), por lo que la acumulación y la fusión de la nieve modulan fuertemente el régimen fluvial. Su influencia es mayor hacia el este debido a la mayor altitud media de las cabeceras (López Moreno y García Ruiz, 2004b). De ahí que el periodo de aguas altas primaverales se desplace desde abril y mayo en la cabecera del río Aragón, hasta junio en los ríos Ara, Cinca, Ésera y Noguera Ribagorzana. El periodo de aguas bajas es claramente estival al oeste e invernal al este (García Ruiz et al., 2001).

\section{Embalses analizados y su régimen de gestión}

La selección de embalses estudiados se basó en la disponibilidad de estaciones meteorológicas cercanas $(<10 \mathrm{~km})$ y en la posibilidad de estimar la superficie inundada por cada embalse en cada momento del año. Así, de algunos importantes embalses pirenaicos como Escales, Canelles, Camarasa, Terradets y Tremp, gestionados por empresas hidroeléctricas, no se ha podido obtener la información necesaria para el cálculo de la superficie inundada. 


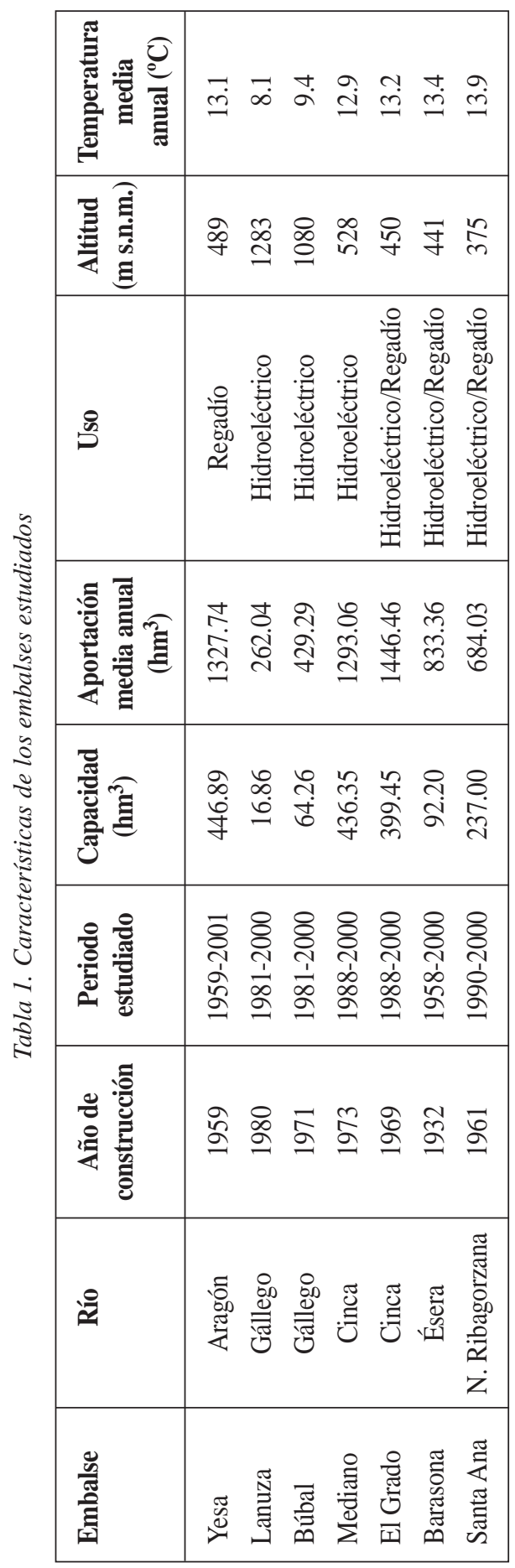


La Tabla 1 resume las características más importantes de los siete embalses analizados. La altitud a la que se encuentra la base de sus presas oscila entre los 375 m s.n.m. de Santa Ana y 1283 m s.n.m. de Lanuza. Yesa es únicamente utilizado para irrigación; Lanuza, Bubal y Mediano están destinados a la producción hidroeléctrica; y a El Grado, Barasona y Santa Ana se les aplica una gestión mixta destinada a compaginar la producción hidroeléctrica con el desvío de agua hacia canales de riego entre los meses de marzo y septiembre. La capacidad de almacenamiento de los embalses oscila entre los 16 y los $447 \mathrm{hm}^{3}$ de Lanuza y Yesa respectivamente. La distinta altitud a la que se encuentran, y por tanto las diferencias en cuanto a régimen fluvial, la demanda que satisfacen (irrigación, hidroelectricidad, uso mixto) y su capacidad de embalsado explican la existencia de patrones de llenado y vaciado muy distintos entre los casos analizados, como se puede observar en la Fig. 2. Así, los embalses que deben derivar agua hacia perímetros de riego poseen una demanda máxima durante verano, por lo que tienden a alcanzar su máximo llenado a finales de la primavera. El periodo de menor volumen almacenado se produce inmediatamente después de la temporada de riego, normalmente en los meses de septiembre y octubre. Aquellos que tienen entre sus funciones producir energía eléctrica poseen también niveles de embalsado elevado durante meses de otoño e invierno, para procurar abastecer la elevada demanda eléctrica invernal. Entre los embalses estudiados existe una notable disparidad de situaciones en cuanto a la variabilidad del volumen embalsado, presentando algunos oscilaciones muy marcadas a lo largo del año (p. ej. Yesa, Búbal, Mediano y Barasona) mientras otros poseen la lámina de agua bastante estable (Lanuza, El Grado y Santa Ana).

\section{Datos y métodos}

La evaporación por unidad de superficie $(\mathrm{mm})$ ha sido calculada mediante la ecuación de Penman para láminas de agua (Penman, 1948), cuya expresión es:

$$
E o=\frac{R n / \frac{\Delta}{\gamma}+E a}{\frac{\Delta}{\gamma}+1}
$$

Donde $E_{o}=$ Evapotranspiración por unidad de superficie; $\Delta=$ Pendiente de la curva; presión de vapor, $\mathrm{kPa}^{\circ} \mathrm{C}^{-1} ; \gamma=$ Constante psicométrica, $\mathrm{kPa}^{\circ} \mathrm{C}^{-1} ; \lambda=$ Calor latente de vaporización, $\mathrm{MJ} \mathrm{kg}^{-1} ; R_{n}=$ Radiación neta, $\mathrm{MJ} \mathrm{m}^{-2} \mathrm{~d}^{-1} ; E a=$ componente aerodinámica y se ha estimado mediante la ecuación de Penman (1963):

$$
E a=0.35\left(0.5+\frac{0.621375 U}{100}\right) 7.500638(e a-e d)
$$




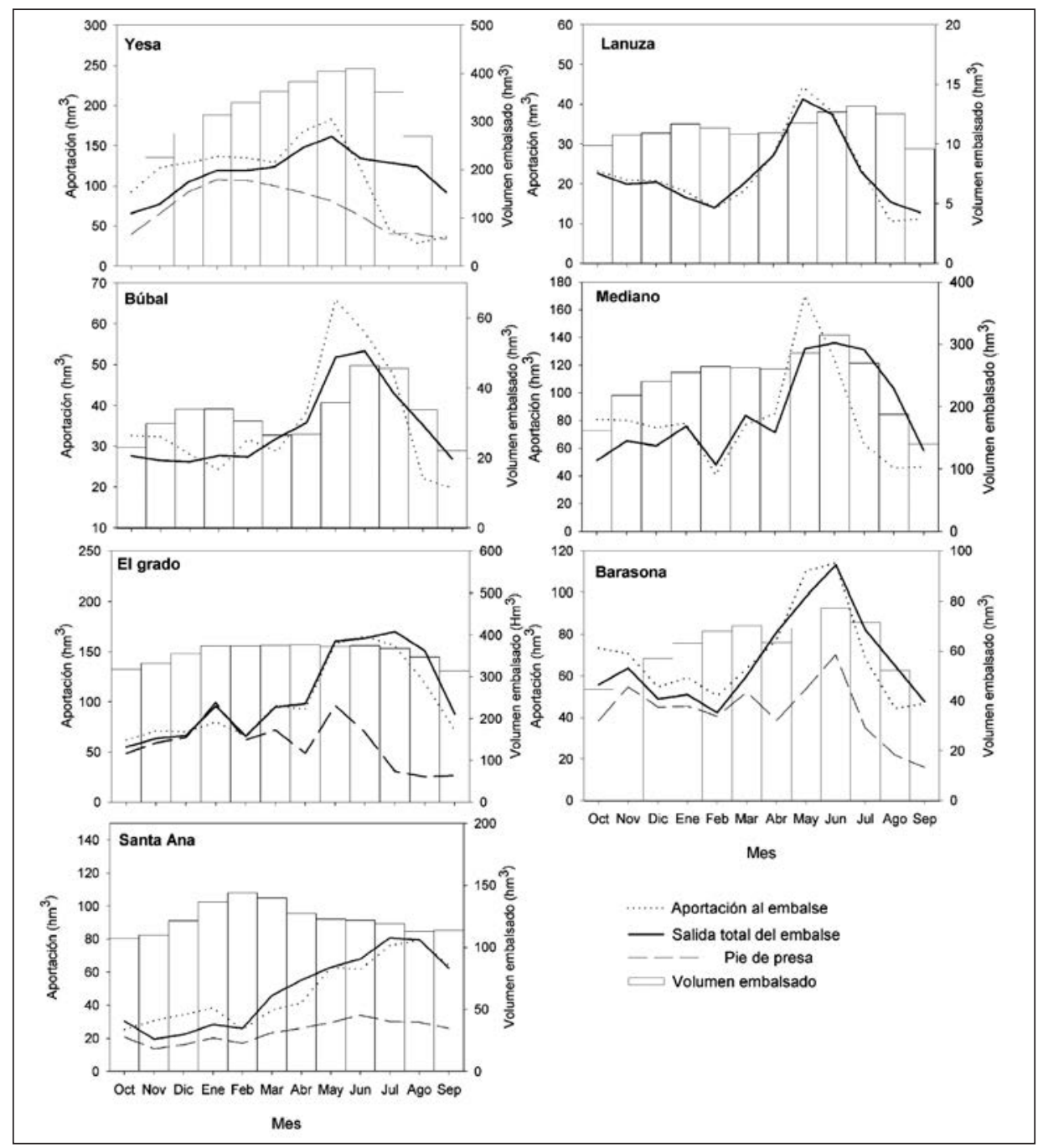

Figura 2. Régimen de gestión (aportación, desembalses y volumen embalsado) en los embalses estudiados

Donde $U$ es la velocidad del viento, ea es la saturación media de la presión de vapor kPa, y ed es la presión de vapor actual, $\mathrm{kPa}$.

Para la aplicación de la ecuación previamente enunciada, se hace necesario disponer además de información térmica, una aproximación a la radiación solar incidente, la humedad relativa y la velocidad del viento. La obtención de los tres últimos parámetros necesarios, a partir de la información básica disponible (temperatura máxima y mínima y precipitación), se ha realizado siguiendo los procedimientos descritos por Allen et al. (1998): 
a) Radiación solar. De acuerdo con la información disponible, la radiación solar fue estimada utilizando la ecuación de Hargreaves y Samani (1985) quienes demostraron que la radiación solar relativa (radiación global de la superficie, Rs) podía considerarse como una porción de la radiación extraterrestre, $R a$, a partir de la temperatura máxima y mínima, mediante:

$$
R_{s}=a R_{a} \sqrt{T_{\max }-T_{\min }}+b
$$

Donde $R s$ es la radiación global en la superficie, $\mathrm{MJm}^{-2} \mathrm{dia}^{-1}$, y $R a$ es la radiación extraterrestre, $\mathrm{MJm}^{-2}$, que se obtiene a partir de la latitud y la fecha utilizando los métodos detallados por Allen et al. (1998). Tmax y Tmin son la temperatura del aire máxima y mínima, ${ }^{\circ} \mathrm{C} ; a$ y $b$ son coeficientes que varían de forma muy progresiva en el espacio y que pueden ser calibrados en localidades donde se cuenta con observaciones, la estación automática de Yesa en este caso.

b) Humedad relativa. Existen numerosas ecuaciones para calcular la humedad relativa a partir de la información térmica, pero probablemente la más utilizada es (Dorenbos y Pruit, 1976):

$$
H r=100 \frac{e T_{d}}{e T_{\text {med }}}
$$

Donde $e T_{d}$ es la presión del vapor en el punto de rocío diario, y $e T_{m e d}$ es el vapor de presión diario obtenido mediante:

$$
e T m e d=\frac{e T_{\max }+e T_{\min }}{2}
$$

siendo

$$
\begin{aligned}
& e\left(T_{\max }\right)=0.6018 e^{\left(\frac{17.27 T_{\max }}{T_{\max }+237.7}\right)} \\
& e\left(T_{\min }\right)=0.6018 e^{\left(\frac{17.27 T_{\min }}{T_{\min }+237.7}\right)}
\end{aligned}
$$

Se asume que el punto de rocío es constante durante el día, y durante la noche equivale a la temperatura mínima (Allen, 1996); si bien algunos estudios indican que en sectores áridos y semiáridos se sitúa 2 ó 3 grados por debajo de la temperatura mínima (Allen, 1996; Hess, 1998).

c) Viento. La velocidad del viento es una variable requerida por la ecuación Penman-Monteith, pero no se dispone de registros continuos fiables en ninguna de las 
estaciones utilizadas en la zona de estudio. No es recomendable extrapolar valores de estaciones cercanas, pues pueden existir importantes diferencias de velocidad entre localidades próximas. Para evaluar el efecto de la falta de información de este parámetro en el cálculo de ETo, ha sido necesario asumir que la velocidad del viento presenta valores similares en toda la cuenca, lo que posiblemente suponga una subestimación en los sectores de alta montaña. Para este trabajo se asumió una velocidad constante de viento de $2 \mathrm{~ms}^{-1}$. Este valor fue seleccionado por: i) ser el recomendado como valor global en caso de ausencia de este tipo de información (obtenido a partir de mediciones en 2.000 localidades) por Allen et al. (1998); y ii) la velocidad media del viento registrada en las estaciones disponibles del Pirineo (periodo 1999-2003) fue de $1.9 \mathrm{~ms}^{-1}$.

Se han calculado valores de evaporación (Eo) para estaciones meteorológicas cercanas a embalses, considerando esos valores representativos de toda la superficie inundada por el embalse. Así, se han calculado valores de Eo para las localidades de Yesa (embalse de Yesa), Sallent de Gállego (embalse de Lanuza), Biescas (embalse de Búbal), Mediano (embalses de Mediano y El Grado), Graus (embalse de Barasona) y Canelles (embalse de Santa Ana).

La evaporación en un embalse no sólo depende del factor climático, puesto que la pérdida de agua total resulta de multiplicar Eo por la superficie inundada en un día o periodo determinado (Mugabe et al., 2003). Como muestra la Fig. 3, para calcular las pérdidas por evaporación de un embalse se calcularon los valores diarios de Eo y superficie inundada, permitiendo conocer la pérdida total del embalse. En el ejemplo presentado (embalse de Yesa, año 1999), puede observarse que tanto el factor climático como el régimen de embalsado interactúan en la explicación de la serie de evolución de pérdidas de agua. Así, la serie de volumen total evaporado muestra sus valores más elevados antes de que se produzcan los mayores valores de evaporación, coincidiendo con el periodo de máximo llenado y por lo tanto de mayor superficie inundada por el embalse. Posteriormente se produce un periodo en el que la evaporación por unidad de superficie es muy elevada, pero el volumen de pérdida total desciende paralelamente a la disminución de la superficie ocupada por la lámina de agua. Igualmente, el descenso de la evaporación en el embalse durante los meses de invierno no aparece representado de una forma tan acusada como en la serie de Eo, pues desde otoño la lámina de agua experimenta un incremento mantenido.

El cálculo de la superficie inundada se realiza mediante la aplicación de polinomios que relacionan la altura de la lámina de agua con la superficie que esta ocupa. Lógicamente estos polinomios varían de forma notable de un embalse a otro y se basan principalmente en la morfología del vaso del embalse. La Tabla 2 muestra los coeficientes de los polinomios utilizados, que fueron proporcionados por la C.H.E. De esta forma la superficie inundada en un día se obtiene mediante la siguiente expresión:

Superficie $=\operatorname{coef} 7 *(\operatorname{cota} \text { día-cotamin. })^{\mathrm{n}}+\operatorname{coef} 6 *(\operatorname{cota} \text { día-cotamin. })^{(\mathrm{n}-1)}+\ldots+$ coef $1 *$ (cota día-cotamin.) +coef0; n=6 en Lanuza, Búbal, Mediano, Barasona y Santa Ana; y $n=7$ en Yesa y El Grado. 


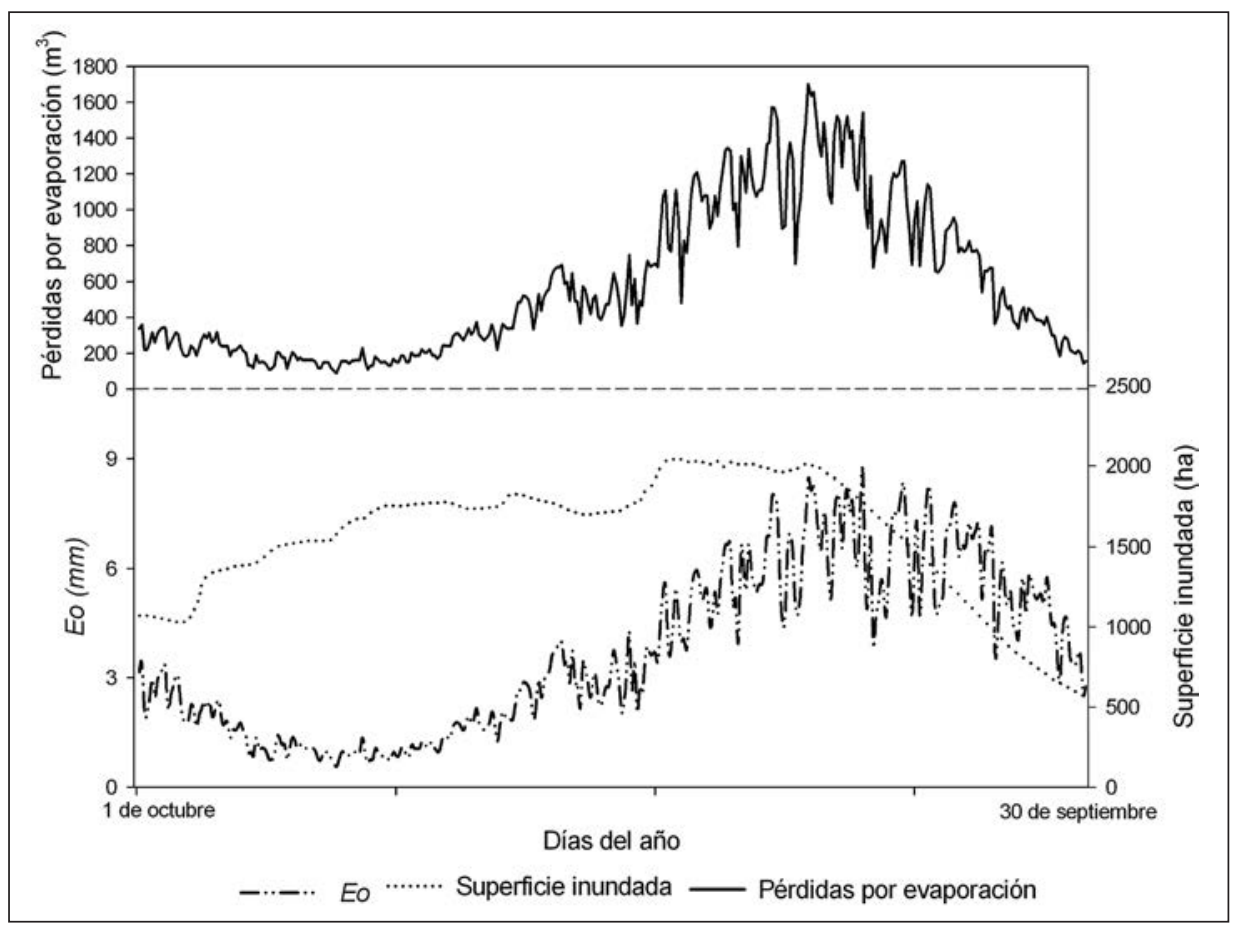

Figura 3. Valores diarios de evaporación estimada (Eo), superficie inundada y pérdida de agua por evaporación en el embalse de Yesa durante el año hidrológico de 1999

La información de la superficie inundada ha sido también utilizada, junto a los datos pluviométricos, para estimar el volumen de precipitación caído sobre la lámina de agua. Su cálculo es importante, pues se trata de un aporte de agua normalmente ignorado en los balances de masa de los embalses, qué pueden compensar en buena parte a las pérdidas ocasionadas por evaporación.

\section{Resultados}

La Fig. 4 muestra los valores medios mensuales de evaporación, pérdida total de agua y superficie inundada en los embalses de Yesa, Lanuza y Búbal. En los tres casos se observa que las pérdidas totales de agua mantienen una evolución semejante a los valores de evaporación estimada, pero con interesantes diferencias que se relacionan con la variabilidad de la superficie inundada a lo largo del año.

En el embalse de Yesa las mayores pérdidas por evaporación se producen en junio y julio, coincidiendo con la mayor tasa de evaporación y una superficie inundada muy elevada. Así, los meses de julio y agosto presentan tasas de evaporación potencial simi- 


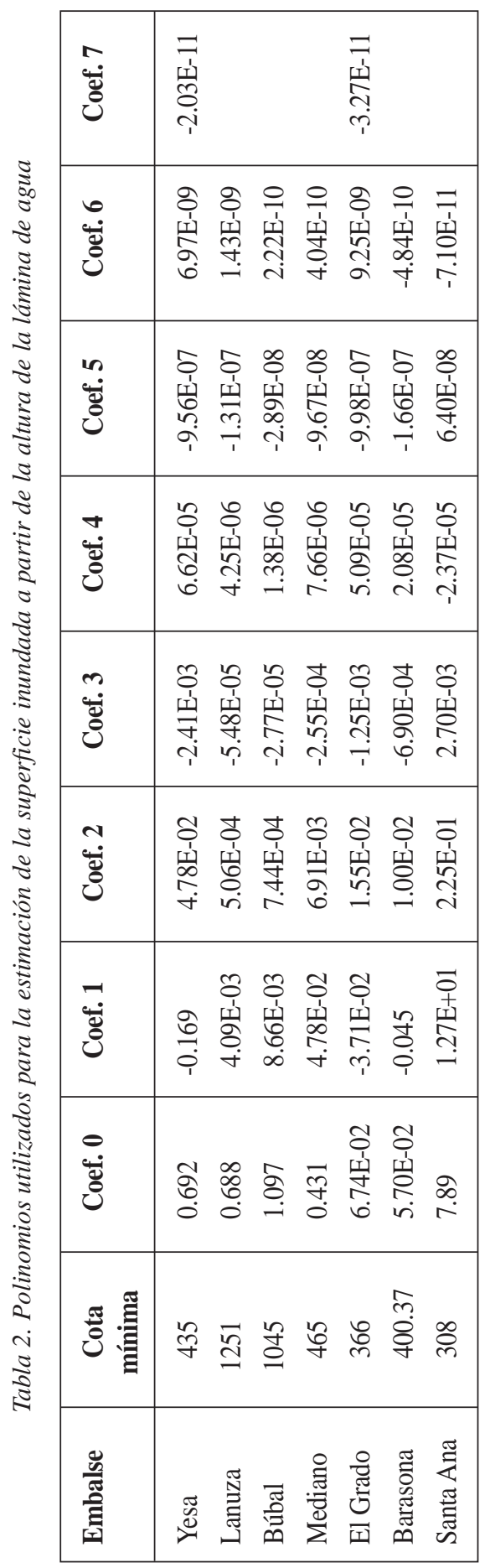


lares e incluso superiores, pero la mayor superficie inundada en junio genera importantes diferencias de pérdidas de volumen total en el embalse ( 3.7 frente a $2.8 \mathrm{hm}^{3} \mathrm{mes}^{-1}$ ). Incluso en mayo se produce una pérdida de agua de $3.1 \mathrm{hm}^{3} \mathrm{mes}^{-1}$, a pesar de que la tasa de evaporación es notablemente inferior a la del mes de agosto. Al igual que se podía observar en la Fig. 3, el importante descenso de evaporación estimada desde septiembre hasta febrero, no se refleja en la evolución de las pérdidas totales en el embalse, que se mantienen constantes debido al progresivo incremento de la lámina inundada.

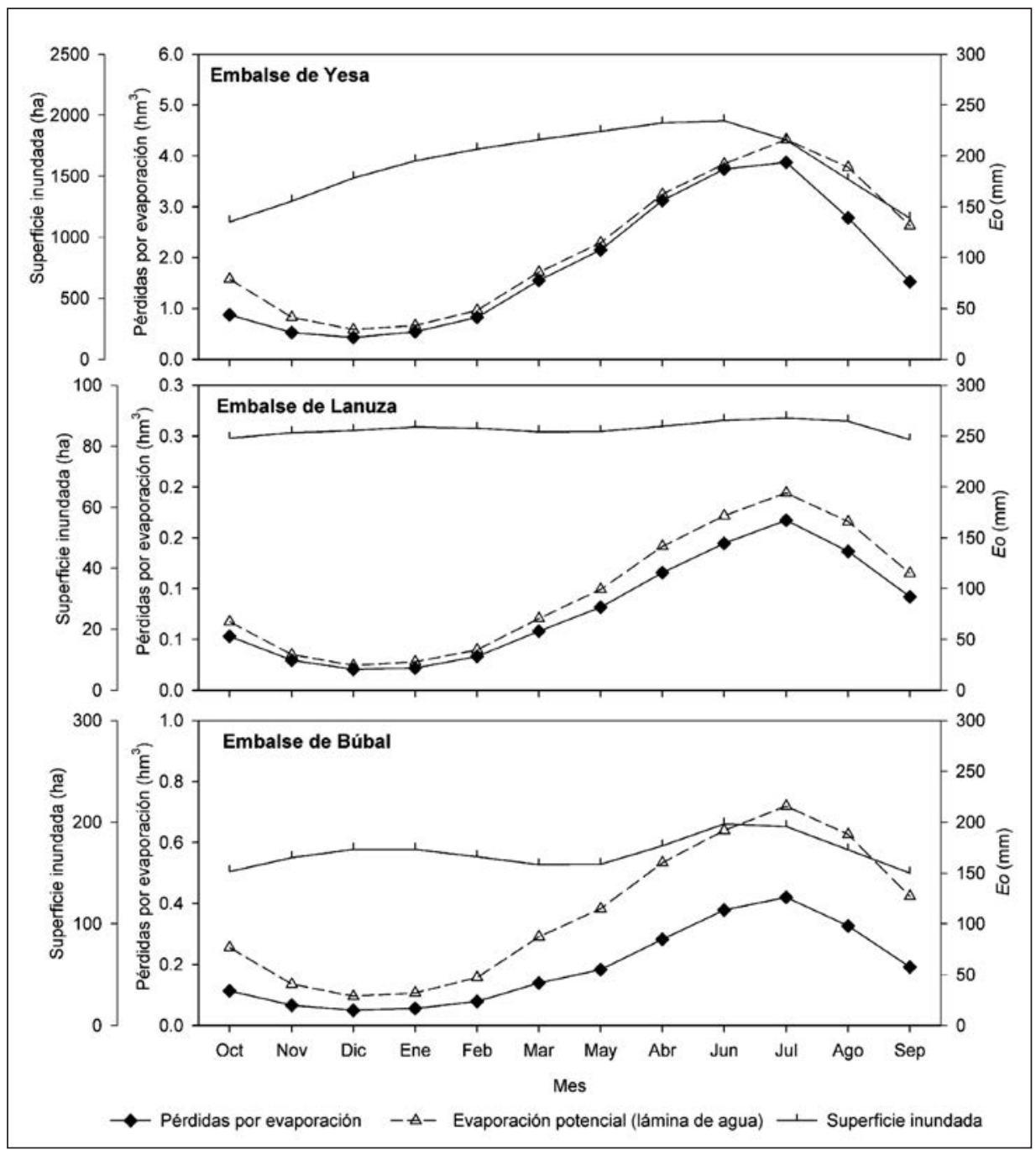

Figura 4. Evaporación estimada (Eo), superficie inundada y pérdida de agua por evaporación media anual en los embalses de Yesa, Lanuza y Búbal 
En el embalse de Lanuza los valores de evaporación y de pérdidas de agua evolucionan a lo largo del año de una forma muy similar. La elevada correspondencia entre ambas curvas se explica porque la superficie inundada se mantiene prácticamente constante a lo largo del año oscilando entre 82.1 y 89.4 ha los meses de menor y mayor superficie respectivamente.

En el embalse de Búbal, la variabilidad estacional de la lámina de agua es mayor que en Lanuza, lo que genera algunas diferencias entre la evolución de los valores de Eo y las pérdidas totales del embalse. Así, como sucedía en Yesa, la mayor superficie inundada durante junio genera pérdidas de agua mayores que en agosto bajo tasas de evaporación muy similares. Al igual que en Yesa, el descenso de Eo en otoño e invierno aparece muy suavizado en respuesta al incremento de superficie inundada a lo largo de este periodo.

La Fig. 5 muestra las mismas variables que la Fig. 4, en este caso para los embalses de Barasona, Mediano, El Grado y Santa Ana. El embalse de Barasona muestra notables fluctuaciones en cuanto a superficie inundada a lo largo del año, que explican las importantes diferencias entre la evolución de Eo y las pérdidas totales producidas por evaporación. Así, las pérdidas estimadas en mayo y junio exceden a las estimadas en agosto, que potencialmente presenta tasas de evaporación más elevadas. Al igual que en otros embalses estudiados, el aumento de superficie inundada entre octubre y febrero, matiza notablemente el efecto del descenso de Eo durante el mismo periodo.

El embalse de Mediano también presenta una elevada oscilación de la superficie inundada, dando lugar a desajustes entre las series de tasa de evaporación y pérdidas totales. De nuevo los meses de mayo, junio y julio son los de mayores pérdidas, reflejándose aún más claramente por el cruce de las dos curvas. Es evidente que a partir de julio el descenso de la evaporación real es superior a la evaporación potencial, dada la rápida contracción espacial de la lámina de agua. El incremento de superficie inundada en otoño es mucho más lento que en el caso de Yesa, Búbal y Barasona y por tanto la evolución de las pérdidas totales es muy similar a la evolución mensual de la tasa de evaporación durante este periodo.

La Tabla 3 muestra un resumen para cada uno de los embalses estudiados indicando los valores anuales de evaporación por unidad de superficie, pérdida anual por evaporación y lo que representa en cuanto al porcentaje de capacidad de almacenamiento y de aportación de caudales al embalse. Se incluye la relación entre tales pérdidas y algunas características de los embalses, como capacidad de embalsado, superficie inundada y la relación entre ambas. Además la tabla presenta la cantidad de precipitación caída directamente sobre la lámina de agua del embalse, lo que permite valorar la importancia de está pérdida, frente a otras entradas de agua que no son normalmente contabilizadas.

Los valores de evaporación por unidad de superficie oscilan entre $1152 \mathrm{~mm}^{2} \tilde{n}^{-1}$ en el embalse de Lanuza y 1413 mm año-1 en el embalse de Barasona. La mayor parte de los embalses muestran valores semejantes, todos por encima de $1300 \mathrm{~mm}$ año ${ }^{-1}$. Sin embargo, las pérdidas de agua por evaporación varían notablemente entre los $22 \mathrm{hm}^{3}$ del 


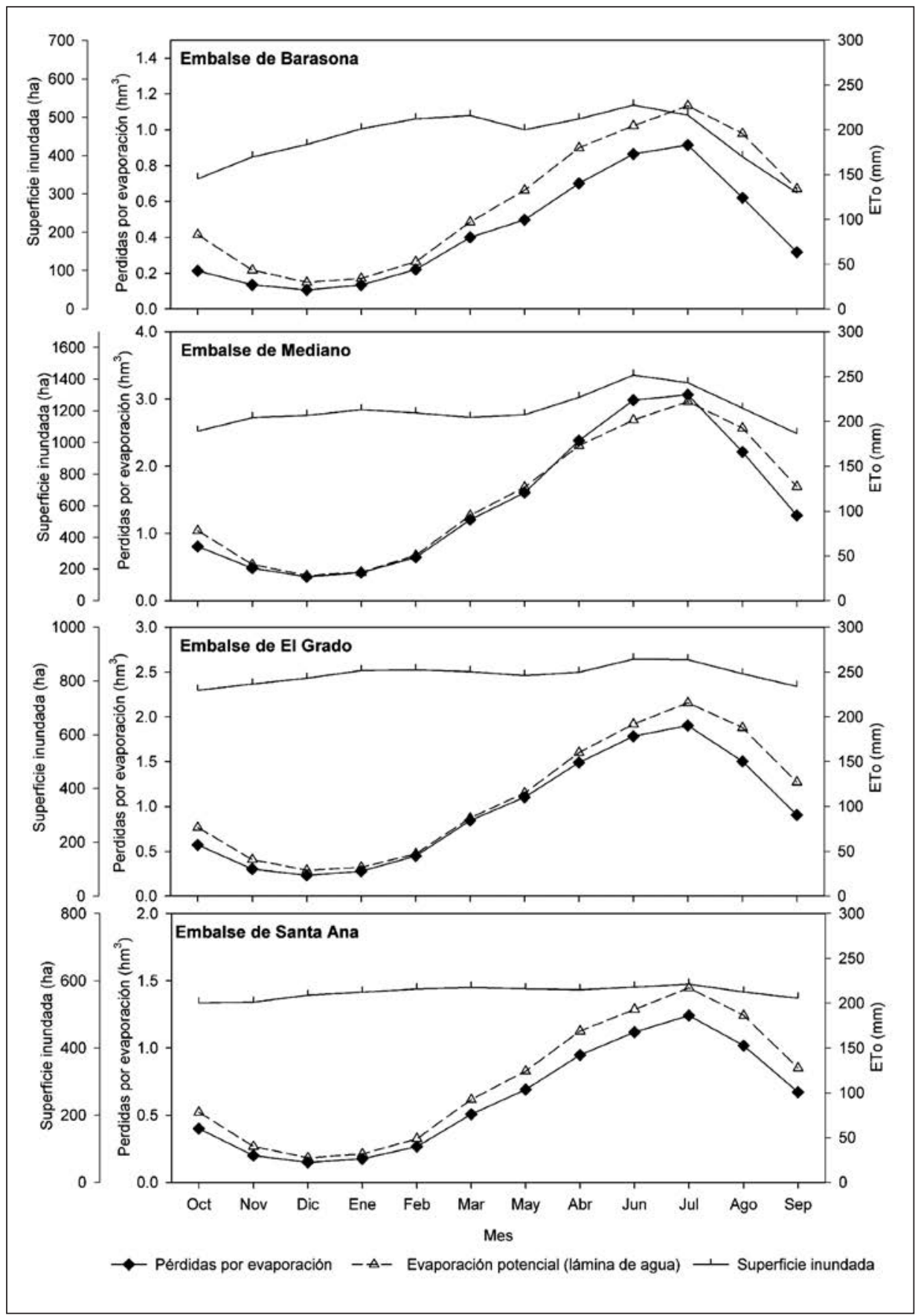

Figura 5. Evaporación estimada (Eo), superficie inundada y pérdida de agua por evaporación media anual en los embalses de Barasona, Mediano, el Grado y Santa Ana 
embalse de Yesa y los $0.9 \mathrm{hm}^{3}$ de Lanuza. Lógicamente existe una clara relación entre las pérdidas de agua por evaporación y el tamaño del embalse. Así, cuando las pérdidas se expresan como un porcentaje de la capacidad de almacenamiento, los valores de pérdida tienen a mostrar diferencias menores, oscilando entre una pérdida del $2.8 \%$ en el embalse de El Grado y un 5.6\% en el de Lanuza. Puede resultar sorprendente que el embalse que tiene una menor evaporación por unidad de superficie (por localizarse a mayor altitud) es el que pierde mayor porcentaje de su volumen. Dos razones explican este hecho: i) el mantenimiento a lo largo de todo el año de una superficie inundada muy elevada, por lo que acusa mayores pérdidas que aquellos embalses cuya lámina muestra una mayor variabilidad estacional; y ii) posee un ratio muy bajo entre capacidad de almacenamiento y superficie inundada (0.18) lo que se traduce en que un mayor porcentaje del volumen total almacenado queda expuesto en la superficie y, por tanto, puede ser evaporado.

En general, los embalses que muestran ratios más bajos entre volumen de agua y superficie, tienden a mostrar porcentajes de pérdida mayores, como sucede en el embalse de Barasona y Yesa (ratios de 0.13 y 0.21 y pérdidas del $5.6 \%$ y $4.9 \%$ respectivamente). En cambio, embalses con elevadas tasas de evaporación anual, pero con una superficie inundada relativamente baja respecto al volumen que almacenan, muestran pérdidas de agua notablemente más bajas, como sucede en los embalses de Santa Ana, Búbal y Mediano (ratios de 0.28, 0.28 y 0.35; y pérdidas del 3.1, 3.6 y $4 \%$ respectivamente). El caso del embalse de El Grado tiene la peculiaridad de que a pesar de tener $399 \mathrm{hm}^{3}$ de capacidad, tan sólo son aprovechables $260 \mathrm{hm}^{3}$. Así, si se considera el volumen total muestra una gran eficacia en cuanto a la relación entre superficie inundada y volumen almacenado, lo que genera una pérdida de un $2.8 \%$ de la capacidad del embalse. En cambio, si se considera únicamente la capacidad útil, el ratio entre volumen embalsado y superficie inundada se reduce a 0.23 y las pérdidas se elevan a un $4.4 \%$, uno de los porcentajes más elevados de los embalses estudiados.

De lo anterior se deduce que en algunos embalses se evaporan volúmenes de agua muy importantes. En algún caso como Yesa o Mediano, la evaporación puede equivaler al volumen total de algunos de los embalses estudiados, como serían los embalses de La Peña o Lanuza. En ciertos meses, las pérdidas por evaporación llegan a suponer una elevada proporción de la aportación que reciben. Así en el embalse de Yesa durante el mes de agosto se evaporan $2.78 \mathrm{hm}^{3}$, un $9.5 \%$ de los $29 \mathrm{hm}^{3}$ que aportan en ese mes los ríos Aragón y Esca al embalse. Sin embargo, si se consideran los valores anuales de forma relativa, en relación a la capacidad de cada embalse o la aportación que les llega, las cifras indican el escaso impacto de este tipo de pérdidas en la gestión del embalse, pues en ningún caso suponen el $6 \%$ de su capacidad ó un $2 \%$ de los caudales que reciben anualmente.

Dependiendo del tamaño de las superficies inundadas por los embalses, la cantidad de agua que llueve directamente sobre la lámina de agua varía entre los 10 y $15 \mathrm{hm}^{3}$, como en Mediano y Yesa, y $1 \mathrm{hm}^{3}$ en Lanuza. Dichos aportes compensan en gran medida las pérdidas por evaporación, reduciéndolas a valores entre 5 y $10 \mathrm{hm}^{3}$ en los gran- 


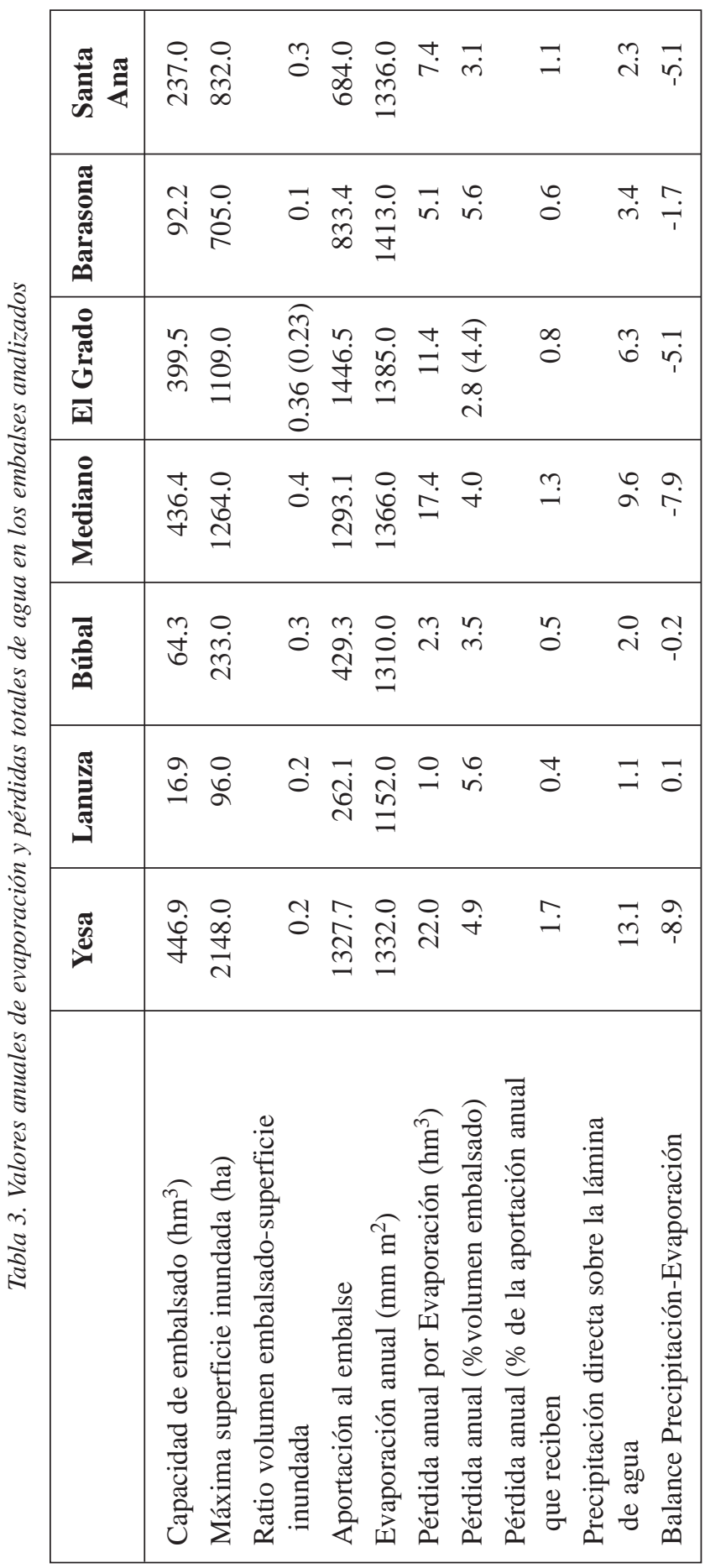


des embalses (p.ej. Yesa, Mediano, El Grado y Santa Ana) situados el sector más meridional y por tanto con escasa precipitación, a situaciones, como el embalse de Lanuza, donde la precipitación excede a las cantidades evaporadas. Este hecho fundamentalmente se explica por la elevada precipitación que registra la zona y por su reducido ratio entre volumen embalsado y superficie inundada.

\section{Discusión y conclusiones}

La estimación de la evaporación potencial diaria para láminas de agua, en combinación con la información de las fluctuaciones de la superficie inundada por los siete embalses analizados, ha permitido realizar una estimación realista de las pérdidas de agua por evaporación en una serie de embalses del Pirineo.

La distinta localización a la que se encuentran los embalses considerados conduce a diferencias en las tasas de evaporación de hasta 261 mm entre los casos más contrastados. La altitud a la que se encuentran los embalses aparenta ser el factor más importante para explicar dicha variabilidad, pues introduce importantes diferencias térmicas, siendo la temperatura el parámetro al que la evaporación se muestra más sensible (Hargreaves y Allen, 2003). La relativamente moderada heterogeneidad en las tasas de evaporación se acentúa de forma muy marcada cuando se cuantifican las pérdidas totales por evaporación que oscilan entre los $22 \mathrm{hm}^{3}$ del embalse de Yesa y los $0.9 \mathrm{hm}^{3}$ de Lanuza. Estas cifras suponen una pérdida de la capacidad total embalsado de entre un $5.6 \%$ y un $2.8 \%$ del volumen embalsado. Los patrones de llenado y vaciado de los embalses y la relación entre capacidad de embalsado y superficie inundada resultan determinantes, superando incluso a los factores climáticos a la hora de explicar la importancia de las pérdidas de recursos hídricos por procesos de evaporación. Así, en varios ejemplos analizados no se han detectado las mayores pérdidas de agua en momentos en que, por razones climáticas, las tasas de evaporación eran más elevadas, sino cuando, por estrategia para la gestión de los embalses, éstos ocupaban la máxima superficie inundada. Es evidente, no obstante, que el destino agrícola de buena parte de los embalses (y más en ambientes mediterráneos o con fuerte estacionalidad pluviométrica y termométrica) hace inevitable que el mayor volumen embalsado se alcance al inicio del verano (o de la estación seca) en un momento en que coinciden una fuerte evaporación y una mayor superficie ocupada por la lámina de agua.

Si bien las cifras proporcionadas en este estudio no apuntan a la evaporación en embalses como un problema clave para la gestión de los recursos hídricos, debe considerarse como una pérdida considerable, pues sólo los siete embalse analizados, que son una pequeña parte de los instalados en la cuenca del Ebro, suman $67 \mathrm{hm}^{3}$, aproximadamente lo que una ciudad como Zaragoza capta anualmente $\left(\approx 70 \mathrm{hm}^{3}\right.$ de captación bruta) para uso doméstico (ver www.ecodes.org). Estos valores pueden ser mucho más elevados para embalses en zonas con mayores tasas de evaporación y menor posibilidad de optimizar la relación volumen embalsado-superficie inundada (p. ej. aquellos situados 
en el tramo bajo del Ebro, o algunos proyectados en el Prepirineo o incluso en el centro de la Depresión). Además, el incremento de las temperaturas, proyectado para la región como consecuencia del cambio climático, puede aumentar notablemente las tasas de evaporación por unidad de superficie y, por tanto, las pérdidas totales de agua (López Moreno et al., 2008 a). Los escenarios de cambio climático indican un incremento medio de las temperaturas anuales de entre 2.8 y $4{ }^{\circ} \mathrm{C}$ según los escenarios de emisión de gases de efecto invernadero, siendo este aumento aún más acusado en los meses de verano (López Moreno et al., 2008 a). Así, un incremento aproximado de entre un 10$20 \%$ en las tasas de evaporación, junto a una situación de mayor stress hídrico y menor disponibilidad de agua prevista para la región (López Moreno et al. 2008 b), puede llevar a que las consecuencias de los procesos evaporativos sobre la disponibilidad de recursos hídricos sea muy superior a la observada en la actualidad.

\section{Agradecimientos}

Este estudio ha sido financiado por los siguientes proyectos: PROBASE CGL200611619 y CANOA CGL 2004-04919-c02-01, ambos financiados por la Comisión de Ciencia y Tecnología, y FEDER. El autor agradece a Sue White y Tim Hess por sus consejos para la estimación de tasas de evaporación en la zona de estudio.

\section{Referencias bibliográficas}

ALLEN, R.G. (1996). Assessing the integrity of weather data for reference evapotranspiration estimation. Agricultural Water Management, ASCE, 18: 159-171.

Allen, R.G., Pereira, L.S., RAES, D. y SMITH, M. (1998). Crop evapotranspiration. Guidelines for computing crop water requirements. Irrigation and Draiange Paper No. 56, FAO, Rome: 300 pp.

AyAla-CARCEDO, F.J. (2000). Impactos del cambio climático sobre los hídricos en España y viabilidad del plan hidrológico. http://tierra.rediris.es/documentos/cambioclima.pdf (comunicación personal).

Batalla, R.J., Kondolf, G.M. y GómEZ, C.M. (2004). Hydrological alterations in the Ebro Basin caused by dams. Journal of Hydrology, 290:117-136.

Bengoechea, J.M., Pérez de los Cobos, J., Pérez Parra, J. y López Segura, J.G. (1991). Evaluación de las pérdidas de agua de riego en el Campo de Dalías, Almería. Symposium sobre el agua en Andalucía. Cordoba. Disponible en: http:// www.larural.es/servagro/sta/publicaciones/riego/pub19707/crt-1-2.htm

FENNESEY, N.M. (1995). Estimating Average Monthly Lake Evaporation in the Noerteast United States. AWRA (Ameriacan Water Resources Association), 36 (4): 759-769. 
García Ruiz, J.M, Puigdefábregas, J. y Creus, J. (1985). Los recursos hídricos superficiales del Alto Aragón. Instituto de Estudios Altoaragoneses, 224 pp., Huesca.

García Ruiz, J.M., Beguería, S., López Moreno, J.I., Lorente, A. y Seeger, M. (2001). Los recursos hídricos superficiales del Pirineo aragonés y su evolución reciente. Geoforma Ediciones, Logroño, $192 \mathrm{pp}$.

HaRgreaVes, G.H. y SAMANI, Z.A. (1985). Reference crop evapotranspiration from temperature. Appl. Eng. Agric, 1 (2): 96-99.

Hargreaves, G.H. y Allen, R. (2003). History and Evaluation of Hargreaves Evapotranspiration Equation. Journal of Irrigation and Drainage Engineering, 129, 1: 5363.

HESS, T.M. (1998). Trends in reference evapo-transpiration in the North East Arid Zone of Nigeria, 1961-1991. Journal of Arid Environments, 38: 99-115.

Higgs, G. y PetTs, G. (1988). Hydrological Changes and River Regulation on the UK. Regulated Rivers: Research and Management, 2: 349-368.

KONDOLF, G.M. (1997). Hungry water: effects of dams and gravel mining on river channels. Environmental Management, 21 (4): 533-551

López Moreno, J.I., BegueríA, S. y GARcía Ruiz, J.M. (2002). Influence of the Yesa reservoir on floods of the Aragón River, central Spanish Pyrenees. Hydrology and Earth System Sciences, 6 (4): 753-762.

López Moreno, J.I., Beguería, S., VAlero-Garcés, B y García Ruiz, J.M. (2003). Intensidad de avenidas y aterramiento de embalses en el Pirineo Central Español. Ería, 61: 159-167.

López Moreno, J.I., BegueríA, S. y GARcíA-Ruiz, J.M. (2004a). The management of a large mediterranean reservoir: storage regimes of the Yesa reservoir, Upper Aragón River basin, Central Spanish Pyrenees. Environmental Management.

LÓPEZ MORENO, J.I. y GARCÍA RUIZ, J.M. (2004b). Influence of snow accumulation and snowmelt on streamflow in the Central Spanish Pyrenees. International Journal of Hydrological Sciences, 49 (5): 787-802.

López Moreno, J.I., García-Ruiz, J.M. y Beniston, M. (2008 a). Environmental Change and water management in the Pyrenees. Facts and future perspectives for Mediterranean mountains. Global and Planetary Change, 66 (3-4): 300-312.

López Moreno, J.I., GoyetTe, S. y Beniston, M. (2008 b). Climate change prediction over complex areas: spatial variability of uncertainties and expected changes over the Pyrenees from a set of regional climate models. International Journal of Climatology, 28 (11): 1535-1550 . 
MAINGI, J.K. y MARSH, S.E. (2002). Quantifying hydrologic impacts following dam construction along the Tana River, Kenia. Journal of Arid Environments, 50: 53-79.

Martínez Álvarez, V., Molina Martínez, J.M. y Soto García, M. (2004). Estimación mediante técnicas SIG, de las pérdidas de agua por evaporación en embalses de riego en la Comunidad de Regantes del Campo de Cartagena. En: Medio Ambiente, Recursos y Riesgos Naturales: Análisis mediante Tecnología SIG y Teledtección, Vol II (Conesa García, C., Álvarez Rogel, Y. y Martínez Guevara, J.B., Edrs.), pp. 37-45, Universidad de Murcia.

MCCUlLy (2001). Silenced Rivers: The ecology and politics of large dams. Zed books. London: $360 \mathrm{pp}$.

MontAseri, M. y AdElOYE, A.J. (2004). A graphical rule for volumetric evaporation loss correction in reservoir capacity-yiel-performanc planning in Urmia region, Irán. Water Resources Management, 18: 55-74.

Mugabe, F.T., Hodnett M.G. y SenZanje, A. (2003). Opportunities for increasing productive water use from dam water: a case study from semi-arid Zimbabwe. Agricultural Water Management, 62: 149-163.

PenMan, H.L. (1948). Natural evaporation from open water, bare soil and grass. Proc. Royal Soc., London, Series A, 193: 120-145.

Penman, H.L. (1963). Vegetation and Hydrology. Tech.Com. nº53. CAB, Farnham, England: $124 \mathrm{pp}$.

PetTS, G.E. (1984). Impounded Rivers. John Willey y Sons Eds. Chichester: 326 pp.

Richter, B.D., Baumgartner, J.V., Wiginton, R. y Braun, D.P. (1997). How much water does a river need?. Freshwater Biology, 37: 231-249.

Sanz Montero, M.E., Avendaño Salas, C. y Cobo Rayan, R. (1999). Influencia de los embalses en el transporte de sedimentos hasta el delta del río Ebro. ManausHydrological Processes in Large Scale River Basins, 1-6.

SÁnchez PÉREZ, J.M. y TREMOLIERES, M. (2003). Change in groundwater chemistry as a consequence of suppression of floods: the case of the Rhine floodplain. Journal of Hydrology, 270: 89-104.

SNOUSSI, M., HAIDA, S. y IMASSI, S. (2002). Effects of the construction of dams on the water and sediment fluxes of the Moulouya and the Sebou rivers. Morocco. Regional Environmental Change, 3: 5-12.

Snyder, N.P., Rubin, D.M., AlPERs, C.N., ChildS, J.R., CurTis, J.A., Flint, L.E. y WRIGHT, S.A. (2004). Estimating accumulation rates and physical properties of sediment behind a dam: Englebright Lake, Yuba River, northern California. Water Resources Research, 40, W11301: 19 pp. 
THOMAS, D.H.L. (1996). Dam construction and ecological change in the riparian forest of the Hadejia-Jama're Floodplain, Nigeria. Land Degradation y Development, 7: 279-295.

Verstraeten, G. y Poesen, J. (2000). Estimating trap efficiency of small reservoirs and ponds. An overview of existing methods and the implications for assessment of sediment yield. Progress in Physical Geography, 24 (2): 219-251.

WAFA, T.A. y LABIB, A.H. (1973). Seepage losses from lake Nasser. En: Man .Mad Lakes: Their Problems and Environmental Effects (Ackermann, W.C., White, G.F. and Worgthington, E.B., Eds). Geophysical Monograph 17, pp. 287-291, American Geophysical Union. Washington, DC, USA.

ZSUFFA, I. (1999). Impact of Austrian hydropower plants on the flood control safety of the Hungarian Danube reach. Hydrological Science Journal, 44, (3): 363-371. 
